\title{
Short-chain fatty acids affect cystic fibrosis airway inflammation and bacterial growth
}

\author{
Peyman Ghorbani ${ }^{1,2,3}$, Prisila Santhakumar ${ }^{1}$, Qingda Hu ${ }^{1,2}$, Pascal Djiadeu ${ }^{1}$, \\ Thomas M.S. Wolever ${ }^{4}$, Nades Palaniyar ${ }^{1,2,5}$ and Hartmut Grasemann ${ }^{1,5,6}$
}

Affiliations: ${ }^{1}$ Program in Physiology and Experimental Medicine, Research Institute, The Hospital for Sick Children, Toronto, ON, Canada. ${ }^{2}$ Dept of Laboratory Medicine \& Pathobiology, University of Toronto, Toronto, ON, Canada. ${ }^{3}$ Institute for Infection Immunology, TWINCORE, Hannover, Germany. ${ }^{4}$ Dept of Nutritional Sciences, University of Toronto, Toronto, ON, Canada. ${ }^{5}$ Institute of Medical Sciences, University of Toronto, Toronto, ON, Canada. ${ }^{6}$ Dept of Pediatrics, Division of Respiratory Medicine, The Hospital for Sick Children and University of Toronto, Toronto, ON, Canada.

Correspondence: Hartmut Grasemann, The Hospital for Sick Children, 555 University Avenue, Toronto, ON M5G 1X8, Canada. E-mail: Hartmut.Grasemanndsickkids.ca

ABSTRACT The hypoxic environment of cystic fibrosis airways allows the persistence of facultative anaerobic bacteria, which can produce short-chain fatty acids (SCFAs) through fermentation. However, the relevance of SCFAs in cystic fibrosis lung disease is unknown. We show that SCFAs are present in sputum samples from cystic fibrosis patients in millimolar concentrations (mean \pm SEM $1.99 \pm 0.36 \mathrm{mM}$ ).

SCFAs positively correlated with sputum neutrophil count and higher SCFAs were predictive for impaired nitric oxide production. We studied the effects of the SCFAs acetate, propionate and butyrate on airway inflammatory responses using epithelial cell lines and primary cell cultures. SCFAs in concentrations present in cystic fibrosis airways $(0.5-2.5 \mathrm{mM})$ affected the release of granulocytemacrophage colony-stimulating factor, granulocyte colony-stimulating factor and interleukin (IL)-6. SCFAs also resulted in higher IL-8 release from stimulated cystic fibrosis transmembrane conductance regulator (CFTR) F508del-mutant compared to wild-type CFTR-corrected bronchial epithelial cells. At $25 \mathrm{mM}$ propionate reduced IL-8 release in control but not primary cystic fibrosis epithelial cells. Low (0.5-2.5 mM) SCFA concentrations increased, while high (25-50 mM) concentrations decreased inducible nitric oxide synthase expression. In addition, SCFAs affected the growth of Pseudomonas aeruginosa in a concentration- and $\mathrm{pH}$-dependent manner.

Thus, our data suggest that SCFAs contribute to cystic fibrosis-specific alterations of responses to airway infection and inflammation.

@ERSpublications

Short-chain fatty acids contribute to CF-specific alterations of responses to airway infection and inflammation http://ow.ly/L7ZI9

This article has supplementary material available from erj.ersjournals.com

Received: Aug 052014 | Accepted after revision: March 302015 | First published online: May 282015

Support statement: This research was supported by the Lynne and Arnold Irwin Foundation and the Cystic Fibrosis Centre at the Hospital for Sick Children (Toronto, ON, Canada; 2014 CF Centre Catalyst Research Grant). N. Palaniyar was supported by Cystic Fibrosis Canada (grant 2619). P. Ghorbani was supported in part by an Ontario Graduate Scholarship. Funding information for this article has been deposited with FundRef.

Conflict of interest: Disclosures can be found alongside the online version of this article at erj.ersjournals.com

Copyright @ERS 2015 


\section{Introduction}

Cystic fibrosis lung disease is characterised by chronic inflammation as well as recurrent and persistent infections of the airways with certain pathogens [1]. Mucus accumulation contributes to inflammation and infection, as well as to sustaining a hypoxic environment [2]. Many typical cystic fibrosis pathogens, including Staphylococcus aureus, Haemophilus influenzae, Streptococcus pneumoniae and Pseudomonas aeruginosa, are facultative anaerobes [3]. Recent advances in metagenomic analysis of the lung microbiome have further revealed a major presence of obligate anaerobic species in cystic fibrosis airways [4, 5], but the clinical relevance of these species in cystic fibrosis lung disease is still unclear.

Metabolism in anaerobic bacteria proceeds in the absence of oxygen. Fermentation of carbohydrates and amino acids results in the formation of short-chain fatty acids (SCFAs) such as acetate $\left(\mathrm{CH}_{3} \mathrm{COO}^{-}\right)$, propionate $\left(\mathrm{C}_{2} \mathrm{H}_{5} \mathrm{COO}^{-}\right)$and butyrate $\left(\mathrm{C}_{3} \mathrm{H}_{7} \mathrm{COO}^{-}\right)$in descending order of abundance. In the large intestine, SCFAs can be found at concentrations ranging from 30 to $150 \mathrm{mM}$ [6]. These metabolites regulate a multitude of functions including intestinal epithelial homeostasis, energy provision, inflammation, regulatory $\mathrm{T}$-cell development and microbial diversity $[7,8]$.

We hypothesised that SCFAs play a role in cystic fibrosis lung disease, specifically in inflammatory responses and host defence against Pseudomonas infection. Our gas chromatography measurements detected millimolar concentrations of SCFAs in sputum obtained from patients with cystic fibrosis. We therefore assessed the effect of SCFAs on inflammation in an alveolar epithelial cell line (A549), cystic fibrosis bronchial epithelial cell lines and primary cell cultures from cystic fibrosis and control donors. We also examined the effect of SCFAs on the growth of $P$. aeruginosa strains. We found that SCFAs at concentrations measured in cystic fibrosis sputum affect the inflammatory responses of airway epithelial cells and the growth of $P$. aeruginosa.

\section{Methods}

\section{Sputum samples}

Sputum samples were obtained from patients with cystic fibrosis as previously described [9] at the Hospital for Sick Children (Toronto, ON, Canada) in accordance with research ethics board approval (The Hospital for Sick Children; 1000019444) [10]. Samples were obtained from clinically stable patients, as well as from patients with a pulmonary exacerbation prior to and following 2 weeks of intravenous antibiotic treatment. See table 1 for patient characteristics.

Solid mucus plugs from sputum samples were processed by treatment with $0.1 \%$ Sputolysin (dithiothreitol, Calbiochem, Etobicoke, ON, Canada) in water [9]. The homogenised sputum was then centrifuged and the supernatant frozen at $-80^{\circ} \mathrm{C}$ for future analysis.

\section{SCFA gas chromatography}

Sputum supernatants were filtered through $30 \mathrm{kDa}$ ultrafiltration devices (Sartorius or Millipore Vivaspin; Sartorius, Mississauga, ON, Canada or Amicon Ultra; Millipore, Etobicoke, ON, Canada). The filtered supernatants were then acidified using formic acid and spiked with an internal standard, 2-methylbutyric acid. Volatile compounds from the acidified filtrates were then distilled under reduced pressure and liquid nitrogen temperatures, dissolved in ultrapure water and injected into a gas chromatography apparatus with flame ionisation detector. Sample SCFA concentrations were calculated based on a standard curve of SCFAs analysed in the same fashion.

\section{TABLE 1 Patient characteristics}

\begin{tabular}{|c|c|c|c|}
\hline & \multirow[t]{2}{*}{ Stable } & \multicolumn{2}{|c|}{ Pulmonary exacerbation } \\
\hline & & $\begin{array}{c}\text { Before antibiotic } \\
\text { treatment }\end{array}$ & $\begin{array}{l}\text { After antibiotic } \\
\text { treatment }\end{array}$ \\
\hline Subjects & 11 & \multicolumn{2}{|c|}{9} \\
\hline Age years & $13.5 \pm 2.0$ & \multicolumn{2}{|c|}{$13.3 \pm 3.4$} \\
\hline Males/females & $6 / 5$ & \multicolumn{2}{|c|}{$5 / 4$} \\
\hline FEV1 \% pred & $87.3 \pm 18.0$ & $52.9 \pm 11.9$ & $63.2 \pm 14.3$ \\
\hline $\begin{array}{l}\text { Predominant bacterial } \\
\text { species }(\mathrm{n})\end{array}$ & $\begin{array}{l}\text { P. aeruginosa (2), } \\
\text { S. aureus ( } 3 \text { ) }\end{array}$ & $\begin{array}{l}\text { P. aeruginosa (3), } \\
\text { S. aureus (4) }\end{array}$ & $\begin{array}{l}\text { P. aeruginosa (1), } \\
\text { S. aureus (3) }\end{array}$ \\
\hline
\end{tabular}

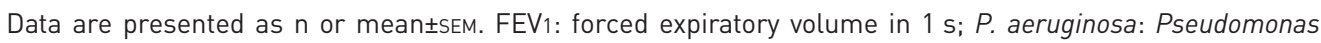
aeruginosa; S. aureus: Staphylococcus aureus. 
Bacterial culture and strains

Bacteria were cultured either from glycerol stocks kept at $-80^{\circ} \mathrm{C}$ or from single colonies selected from trypticase soy agar plates. P. aeruginosa strains PAO1 and PA508 (a gift from the laboratory of Neil B. Sweezey, The Hospital for Sick Children) were cultured in trypticase soy broth (TSB) at $37^{\circ} \mathrm{C}$ for all experiments. Bacteria were grown overnight in TSB at $37^{\circ} \mathrm{C}$ and $225 \mathrm{rpm}$ shaking, and further diluted in TSB modified with different concentrations of SCFAs or other chemicals (sodium acetate, sodium propionate, sodium butyrate and sodium L-lactate) and adjusted to $\mathrm{pH} 7.0,6.5,6.0$ and 5.5 with hydrochloric acid. Microplates were read on an Omega plate reader (BMG Labtech, Cary, NC, USA) for optical density at $600 \mathrm{~nm}$. For analysis, blank TSB-corrected optical density readings were normalised to $\mathrm{pH}$-matched TSB controls for each time point. For microaerobic conditions, plates were sealed using optically clear PCR film at the start of the experiment. Due to condensation on the film, optical density was read at $600 \mathrm{~nm}$ and subtracted by optical density at $800 \mathrm{~nm}$.

\section{Cell lines and culture}

A549 human adenocarcinomic alveolar basal epithelial cells, cystic fibrosis bronchial epithelial 41o-immortalised cells from a patient with homozygous F508del cystic fibrosis transmembrane conductance regulator (CFTR) alleles transfected with F508del (CFBE) or wild-type CFTR vector (corrCFBE) were used in this work. A549 and CFBE cells were cultured in 10\% v/v fetal bovine serum in DMEM (Wisent Bioproducts, St Bruno, QU, Canada) with $4.5 \mathrm{~g} \cdot \mathrm{L}^{-1}$ glucose, $110 \mathrm{mg} \cdot \mathrm{L}^{-1}$ sodium pyruvate, $2 \mathrm{mM}$ L-glutamine, 100 units $\cdot \mathrm{mL}^{-1}$ penicillin/streptomycin at $37^{\circ} \mathrm{C}$ and $5 \% \mathrm{CO}_{2}$. CFBE and corrCFBE cells were cultured with $300 \mu \mathrm{g} \cdot \mathrm{mL}^{-1}$ hygromycin B.

Primary human bronchial epithelial cells were obtained from control or cystic fibrosis donors from the Primary Airway Cell Biobank (McGill University, Montreal, QU, Canada) and cultured in bronchial epithelial growth medium. Human bronchial epithelial cells were cultured in bronchial epithelial growth medium containing $10 \%$ bovine serum albumin, $50 \mu \mathrm{g} \cdot \mathrm{mL}^{-1}$ gentamycin, $100 \mathrm{units} \cdot \mathrm{mL}^{-1}$ penicillin, $100 \mu \mathrm{g} \cdot \mathrm{mL}^{-1}$ streptomycin and $250 \mu \mathrm{g} \cdot \mathrm{mL}^{-1}$ amphotericin B. Cells were passaged once before reaching $80-90 \%$ confluence and thereafter cultured until $90 \%$ confluence and subsequently used for the experimental procedure.

\section{Cytomix stimulation}

A549, CFBE, corrCFBE or primary human bronchial epithelial cells were seeded in 12- or 24-well plates and grown to $70-90 \%$ confluence. Plates were then washed, replenished with serum-free media and incubated for $24 \mathrm{~h}$. Cells were then incubated for $1 \mathrm{~h}$ with various SCFAs prior to cytomix $\left(10 \mathrm{ng} \cdot \mathrm{mL}^{-1}\right.$ each of interleukin (IL)- $1 \beta$, tumour necrosis factor (TNF)- $\alpha$ and interferon (IFN) $-\gamma$ ) stimulation and further incubation for specified times. SCFA concentrations were chosen based on ranges found in the colonic lumen (10-50 $\mathrm{mM})$ and in cystic fibrosis airways $(0.1-5 \mathrm{mM})$, and from previous studies.

\section{Western blot}

Samples were electrophoresed and transferred onto nitrocellulose membranes. Membranes were incubated with anti-inducible nitric oxide synthase (iNOS) or anti-glyceraldehyde 3-phosphate dehydrogenase antibodies (Santa Cruz Biotechnology, Dallas, TX, USA) and anti-rabbit horseradish peroxidase conjugated secondary antibodies (Santa Cruz). Enhanced chemiluminescent substrate (Thermo, Mississauga, ON, Canada) was added directly onto each membrane and developed using radiographic film on a Kodak X-Omat 2000a processor (Windsor, CO, USA).

\section{Cytokine analysis}

Cell culture supernatants were analysed for cytokines using a Milliplex kit (Millipore) according to the manufacturer's protocol. IL-8 concentrations were measured using a commerical ELISA kit (PeproTech, Rocky Hill, NJ, USA).

\section{Proliferation/viability assays}

For proliferation assays, cells were seeded at $5 \times 10^{4} \mathrm{cells} \cdot \mathrm{mL}^{-1}$ in 96 -well plates and incubated for $1 \mathrm{~h}$ with SCFAs, then incubated for $24 \mathrm{~h}$ with PrestoBlue reagent according to the manufacturer's protocol (Molecular Probes, Burlington, ON, Canada). To assess viability, cells were grown to $80-90 \%$ confluence in 96-well plates before incubation with SCFAs.

\section{Statistical procedures}

Statistical analysis was performed using Microsoft Excel (Redmond, WA, USA) or GraphPad Prism 5 (La Jolla, CA, USA). For sputum SCFAs, unpaired and paired nonparametric t-tests were performed, as well as linear regressions for correlation analyses. For bacterial growth data, repeated measures ANOVA were performed followed by Dunnett's post-test to compare growth curves against the buffer control. For all 
others, unpaired nonparametric t-tests were performed. p-values $<0.05$ were regarded as statistically significant for all tests.

\section{Results}

SCFAs in sputum of patients with cystic fibrosis

SCFAs were found in cystic fibrosis patient sputum in millimolar concentrations (fig. 1a). In clinically stable subjects, mean (range) total SCFA concentration was $1991 \mu \mathrm{M}(821-4064 \mu \mathrm{M})$. For patients presenting with a cystic fibrosis pulmonary exacerbation, mean (range) total SCFA concentrations before antibiotic treatment and after 2 weeks treatment with i.v. antibiotics were $1280 \mu \mathrm{M}(158-4570 \mu \mathrm{M})$ and $873 \mu \mathrm{M}(166-4178 \mu \mathrm{M})$, respectively (fig. 1a). Mean total SCFA concentrations were significantly lower after treatment when compared to stable patients ( $\mathrm{p}=0.0076$ using Mann-Whitney U-test). Although SCFA concentrations decreased for the majority (seven out of nine) of patients after antibiotic treatment, this decrease was statistically nonsignificant (fig. 1a) ( $n=9, p=0.2031$ using Wilcoxon paired test).

The number of neutrophils in sputum is an established marker of cystic fibrosis airways inflammation. Sputum neutrophil counts showed a significant positive correlation with total SCFAs in stable patients (fig. 1b) $\left(\mathrm{n}=10, \mathrm{r}^{2}=0.8743, \mathrm{p}<0.0001\right)$, but not in patients with pulmonary exacerbation, either before $\left(n=9, r^{2}=0.0055, p=0.85\right)$ or after antibiotic treatment $\left(n=9, r^{2}=0.1999, p=0.2276\right)$.

Airway nitric oxide (NO) has previously been shown to be decreased in cystic fibrosis and both exhaled $\mathrm{NO}$ and sputum oxides of nitrogen $\left(\mathrm{NO}_{\mathrm{x}}\right)$ increase after antibiotic treatment for cystic fibrosis pulmonary exacerbations $[11,12]$. Total SCFAs in patients before treatment with antibiotics correlated negatively with the fold change in sputum $\mathrm{NO}_{\mathrm{x}}$ following antibiotic treatment (fig. 1c) $\left(\mathrm{n}=7, \mathrm{r}^{2}=0.7493, \mathrm{p}<0.05\right.$ ), suggesting that high SCFA concentrations in sputum prior to treatment with antibiotics prevented the expected increase in airway NO production. However, there was no correlation between SCFAs and fold change in sputum IL-8 concentrations following antibiotic treatment (fig. 1d) ( $\left.\mathrm{n}=9, \mathrm{r}^{2}=0.0004, \mathrm{p}=0.9605\right)$.

\section{SCFAs modulate inflammatory responses of airway epithelial cells}

SCFAs have been shown to exert pro- or anti-inflammatory effects on intestinal epithelial cells, depending on their concentration [13-16]. To investigate the effects of SCFAs on inflammatory responses of airway and alveolar epithelial cells, we stimulated cells with a mix of IL- $1 \beta$, IFN- $\gamma$ and TNF- $\alpha$ after SCFA pretreatment. Predominant cytokines secreted by A549 alveolar basal epithelial cells were granulocyte-macrophage colony-stimulating factor (GM-CSF), IL-1 $\alpha$, IL-6 (fig. 2a-c) and IL-8 (data not shown). Pre-incubation with SCFAs upregulated the production of GM-CSF and IL- $1 \alpha$, and less so IL-6, in a concentration-dependent manner. Interestingly, while all SCFAs at $0.5-5 \mathrm{mM}$ upregulated GM-CSF and IL-1 $\alpha$ production, acetate at $25 \mathrm{mM}$ resulted in upregulated GM-CSF, IL-1 $\alpha$ and IL-6 (fig. 2a-c).
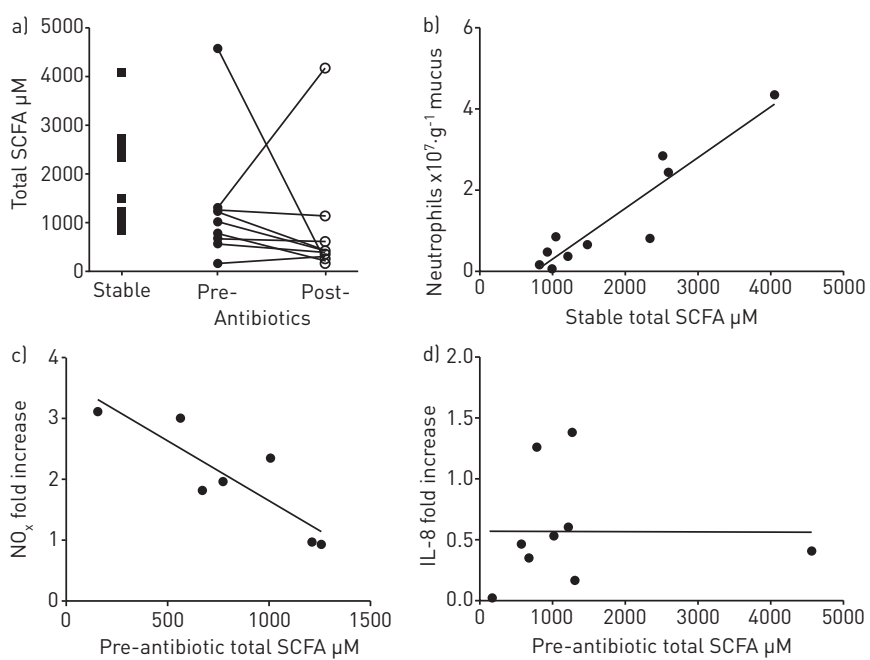

FIGURE 1 a) Total (sum of acetate, propionate and butyrate) concentrations of short-chain fatty acids (SCFAs) in sputum from patients with stable cystic fibrosis lung disease or with a pulmonary exacerbation before and after 14 days of intravenous antibiotic treatment. b) Correlation of total SCFA concentration to neutrophil numbers in patient sputum. Correlation of total SCFA concentrations in patients before antibiotic treatment to c) the fold change in oxides of nitrogen $\left(\mathrm{NO}_{\mathrm{x}}\right)$ or d) the fold change in interleukin (IL)-8 after antibiotic treatment. 

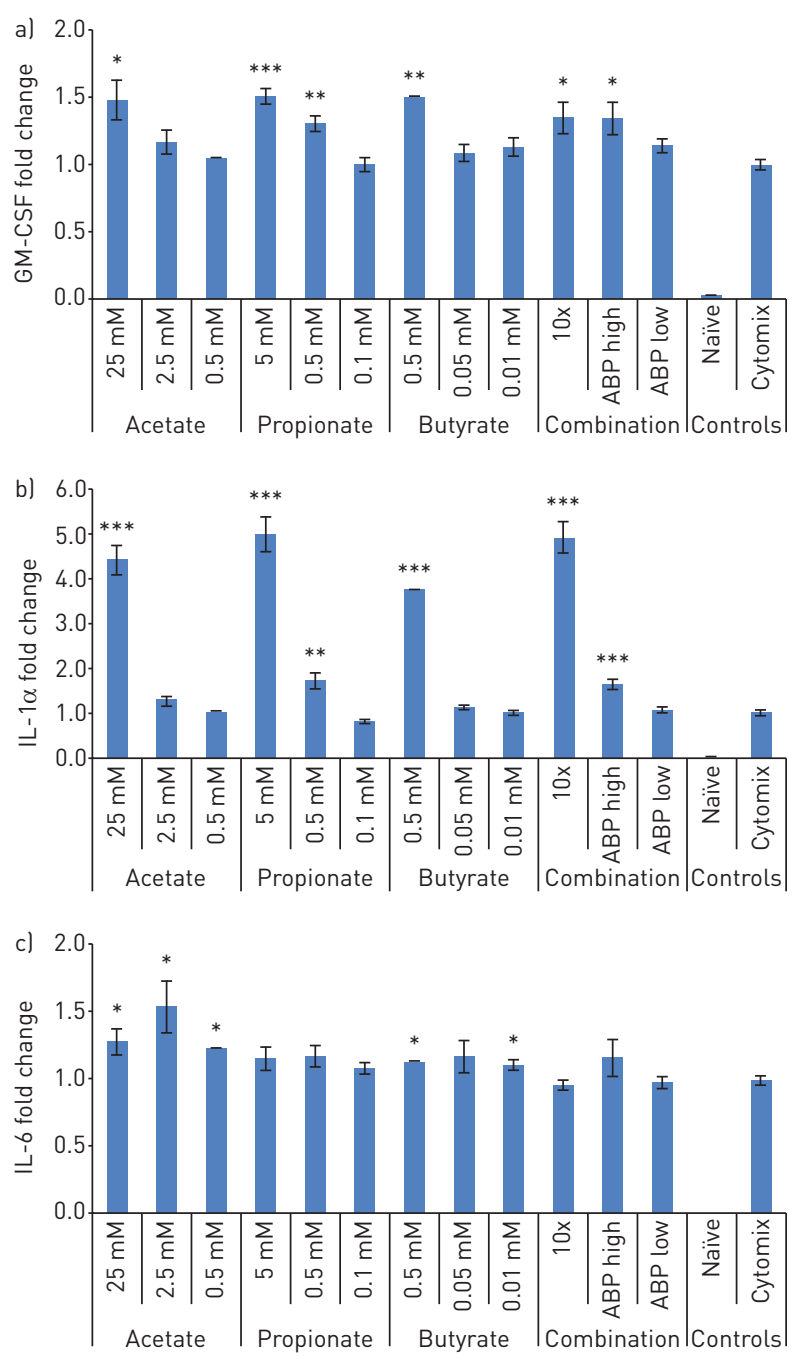

FIGURE 2 Short-chain fatty acids (SCFAs) modulate cytokine secretion in A549 alveolar epithelial cells in response to SCFA incubation and cytokine stimulation. Secretion of a) granulocyte-macrophage colony-stimulating factor (GMCSF); b) interleukin (IL)-1 $\alpha$; and c) IL-6 are shown relative to cytokine stimulation alone (cytomix: $10 \mathrm{ng} \cdot \mathrm{mL}^{-1}$ each of IL-1 $\beta$, tumour necrosis factor- $\alpha$ and interferon- $\gamma$ ). Data are presented as mean \pm SEM. $10 \mathrm{x}: 25 \mathrm{mM}$ acetate, $5 \mathrm{mM}$ propionate and $0.5 \mathrm{mM}$ butyrate; APB high: $2.5 \mathrm{mM}$ acetate, $0.5 \mathrm{mM}$ propionate and $0.05 \mathrm{mM}$ butyrate; APB low: $0.5 \mathrm{~mm}$ acetate, $0.1 \mathrm{mM}$ propionate and $0.005 \mathrm{mM}$ butyrate. ${ }^{*}: p<0.05 ;{ }^{* *}: p<0.01 ;{ }^{* *}: p<0.001$ (nonparametric unpaired t-tests).

Cystic fibrosis airways exhibit a persistent inflammatory environment and several cytokines including GM-CSF, IL-1, IL-6, IL-8, IFN- $\gamma$ and TNF- $\alpha$ are present in cystic fibrosis airways in high concentrations $[17,18]$. Therefore, we next examined the impact of SCFAs on the inflammatory profile of CFBE cells [19], the most common cystic fibrosis-causing mutation in Caucasians [1], transfected with a vector overexpressing F508del-CFTR. As a control we used the same cell line transfected with a vector overexpressing wild-type CFTR (corrCFBE) [20]. Similar to the results in A549 cells, SCFAs at 0.5-2.5 mM, in particular propionate, increased GM-CSF, and granulocyte (G)-CSF production in cytokine-stimulated corrCFBE cells, but not in CFBE cells (fig. 3a and c), while propionate upregulated IL-6 in both cell lines (fig. 3b). Acetate at $0.5 \mathrm{mM}$ reduced IL-6 expression in CFBE but not corrCFBE cells. A mix of acetate $(0.5 \mathrm{mM})$, propionate $(0.1 \mathrm{mM})$ and butyrate $(0.005 \mathrm{mM}$ ) also resulted in increased IL- 6 and G-CSF in the corrCFBE cells only (fig. $3 \mathrm{~b}$ and c, APB low). In contrast, the production of IL-8, a key cytokine for granulocyte recruitment found in high concentrations in cystic fibrosis airways, was upregulated in the presence of $0.5-2.5 \mathrm{mM}$ acetate in stimulated CFBE but not in corrCFBE cells (fig. 3d).

In primary airway epithelial cells, we found that high $(25 \mathrm{mM})$ propionate concentrations downregulated GM-CSF, IL-6 and G-CSF in both cystic fibrosis and non-cystic fibrosis control cells with or without cytomix stimulation (fig. $4 \mathrm{a}-\mathrm{f})$. In contrast, low $(0.5 \mathrm{mM})$ propionate concentrations upregulated G-CSF in stimulated cystic fibrosis but not control cells (fig. 4f), in contrast to the findings in the CFBE cells. We also observed 

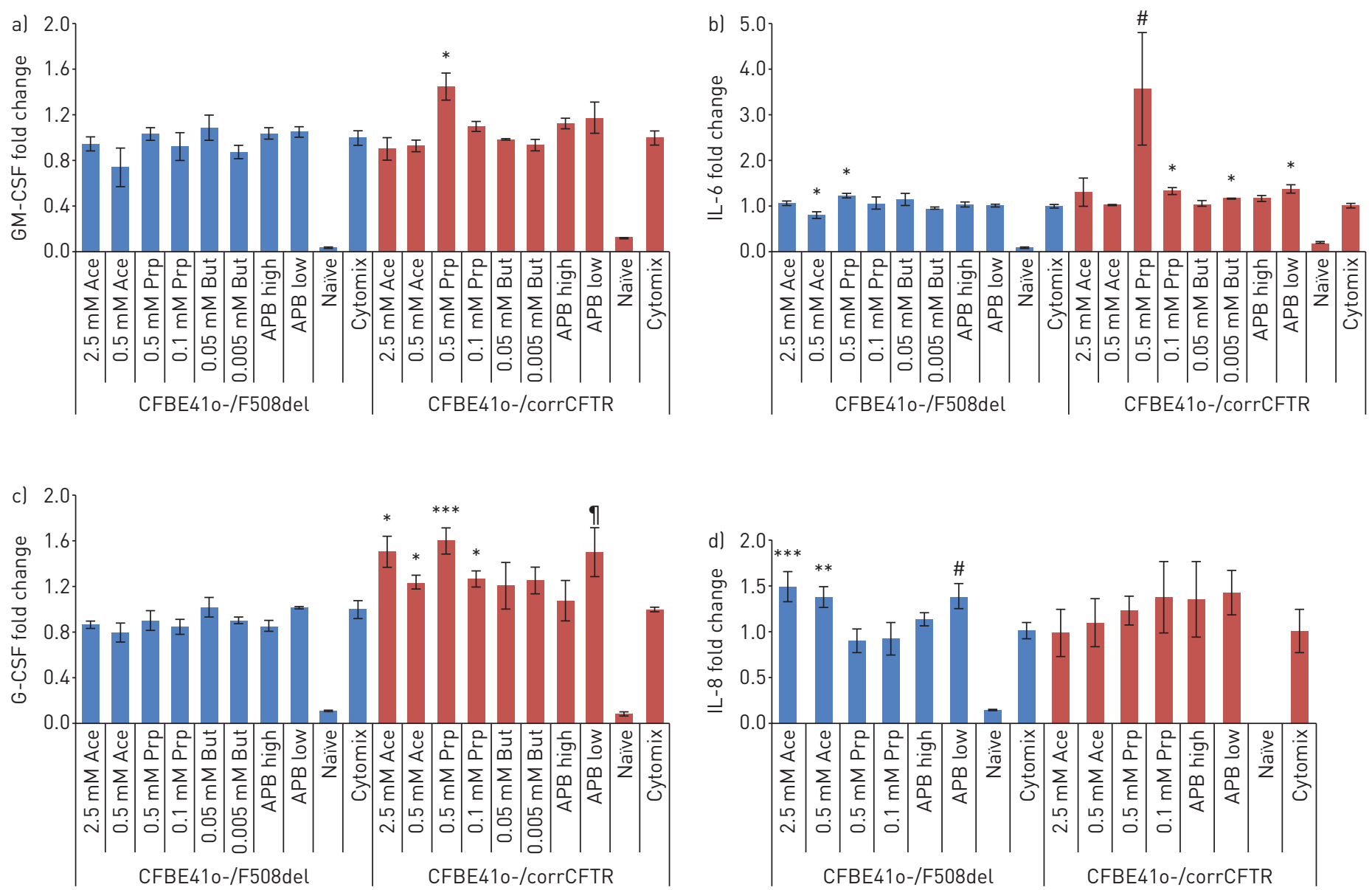

FIGURE 3 Short-chain fatty acids (SCFAs) modulate cytokine secretion in cystic fibrosis bronchial epithelial cells. Bronchial epithelial 41o-immortalised cells from a patient with homozygous F508del cystic fibrosis transmembrane conductance regulator (CFTR) alleles transfected with F508del (CFBE410-/F508del) or wild-type CFTR vector (CFBE410-/corrCFTR) cell lines in response to SCFA incubation and stimulation with cytomix $\left(10 \mathrm{ng} \cdot \mathrm{mL}^{-1}\right.$ each of interleukin (IL)-1 $\beta$, tumour necrosis factor- $\alpha$ and interferon- $\left.\gamma\right)$ for $24 \mathrm{~h}$. Secretion of a) granulocyte-macrophage colony-stimulating factor (GM-CSF); b) IL-6; c) granulocyte-colony-stimulating factor; and d) IL-8 are shown relative to cytokine stimulation alone (cytomix). Data are presented as meanıSEM. Ace: acetate; Prp: propionate; But: butyrate; APB high: $2.5 \mathrm{mM}$ acetate, $0.5 \mathrm{mM}$ propionate and $0.05 \mathrm{mM}$ butyrate; APB low: $0.5 \mathrm{~mm}$ acetate, $0.1 \mathrm{mM}$ propionate and $0.005 \mathrm{mM}$ butyrate. ${ }^{*}: p<0.05{ }^{* *}: p<0.01 ;{ }^{* * *}: p<0.001 ;{ }^{\#}: p=0.10 ;{ }^{9}: p=0.08$ (nonparametric unpaired t-tests). 

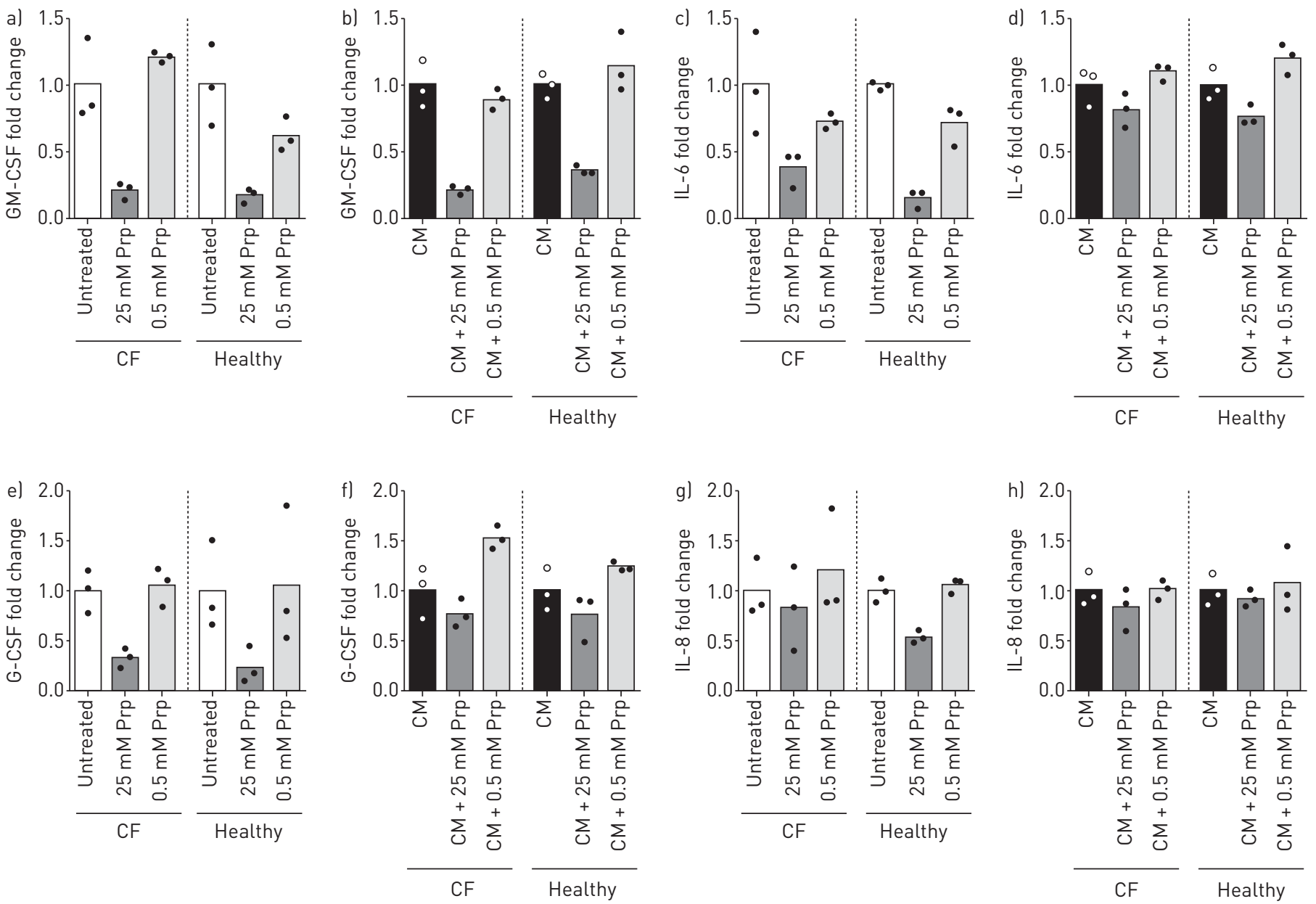

FIGURE 4 Short-chain fatty acids (SCFAs) alter cytokine release in untreated but not stimulated primary epithelial cultures. a, b) Granulocytemacrophage colony-stimulating factor (GM-CSF); c, d) interleukin (IL)-6; e, f) granulocyte (G)-CSF; or g, h) IL-8 expression in primary cystic fibrosis (CF) or healthy control epithelial cells pre-incubated for $1 \mathrm{~h}$ with different concentrations of propionate ((Prp) $0.5 \mathrm{mM}$ and $25 \mathrm{mM}$ ) and $a, c, e, g)$ untreated or $b, d, f, h)$ stimulated with cytomix (CM) (10 ng. $\mathrm{mL}^{-1}$ each of interferon- $\gamma$, tumour necrosis factor- $\alpha$ and IL-1 $\beta$ ) for $24 \mathrm{~h}$. Data are presented as individual replicates (points) and means (bars). Expression is shown relative to untreated or cytomix controls, respectively.

that $25 \mathrm{mM}$ propionate reduced IL-8 in primary control but not cystic fibrosis cells, in the absence of cytokine stimulation (fig. 4g). SCFAs did not affect IL-8 production with cytokine stimulation in either control or cystic fibrosis cells, possibly due to the high basal IL-8 levels following cytokine stimulation (fig. 4h).

Next we examined epithelial cells for iNOS expression under inflammatory cytokine stimulation. Interestingly, low concentrations (0.5-2.5 mM) of SCFAs increased iNOS expression in A549 cells above cytokine stimulation alone at early ( $4 \mathrm{~h}$ and $8 \mathrm{~h}$ ) (online supplementary figs S1a and b and S2a and b) and late (24 h) time points (fig. 5a-c and figs S1c and S2c). In contrast, at higher concentrations (25-50 mM), similar to those previously reported in the intestines of healthy subjects, individual SCFAs or SCFAs in combination significantly reduced cytokine-induced iNOS expression (fig. 5 and figs S1 and S2). The expression of iNOS was absent in unstimulated A549 cells and was not upregulated by butyrate (fig. 5) or acetate/propionate alone (data not shown). We were unable to detect iNOS in the transfected cystic fibrosis cell lines (CFBE or corrCFBE) before or after cytokine stimulation, in line with previous reports [21]. In primary cultures of non-cystic fibrosis airway epithelial cells, expression of iNOS in response to SCFA incubation and cytokine stimulation was similar to A549 cells (fig. 6). In stimulated primary cystic fibrosis epithelial cells, iNOS protein expression was detectable but was reduced in comparison with control cells. Importantly, and in line with the findings in A549 and non-cystic fibrosis control cells, expression of iNOS in cystic fibrosis primary cells was further reduced to undetectable levels with high $(25 \mathrm{mM})$ propionate treatment, but low $(0.5 \mathrm{mM})$ propionate resulted in increased iNOS expression (fig. 6).

Thus, our data suggest that SCFAs mediate important changes in epithelial cell inflammatory cytokine production and iNOS expression, and that cystic fibrosis epithelial cells respond differently to SCFA exposure than do non-cystic fibrosis cells. 
a)

4 h stimulation

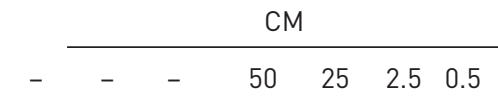

Butyrate $\mathrm{mM}$
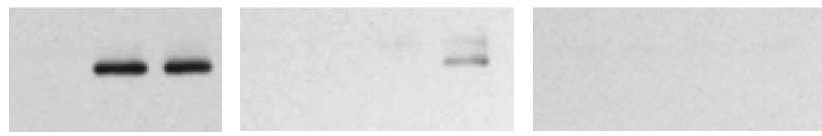

iNOS
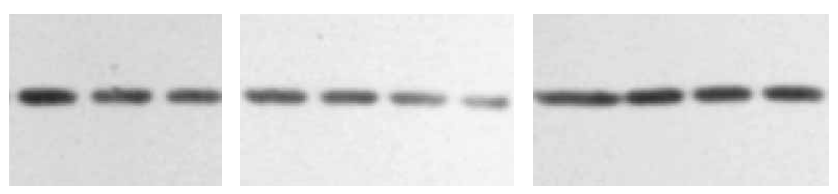

GAPDH

b)

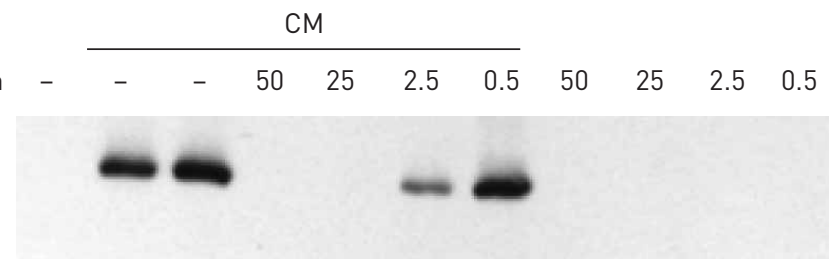

Butyrate mM

$8 \mathrm{~h}$ stimulation

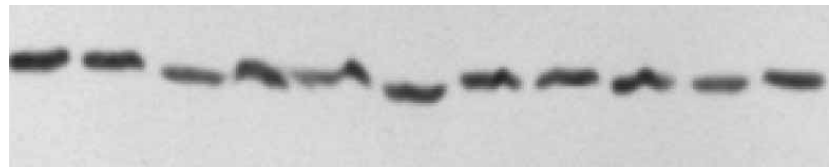

iNOS

GAPDH

c)
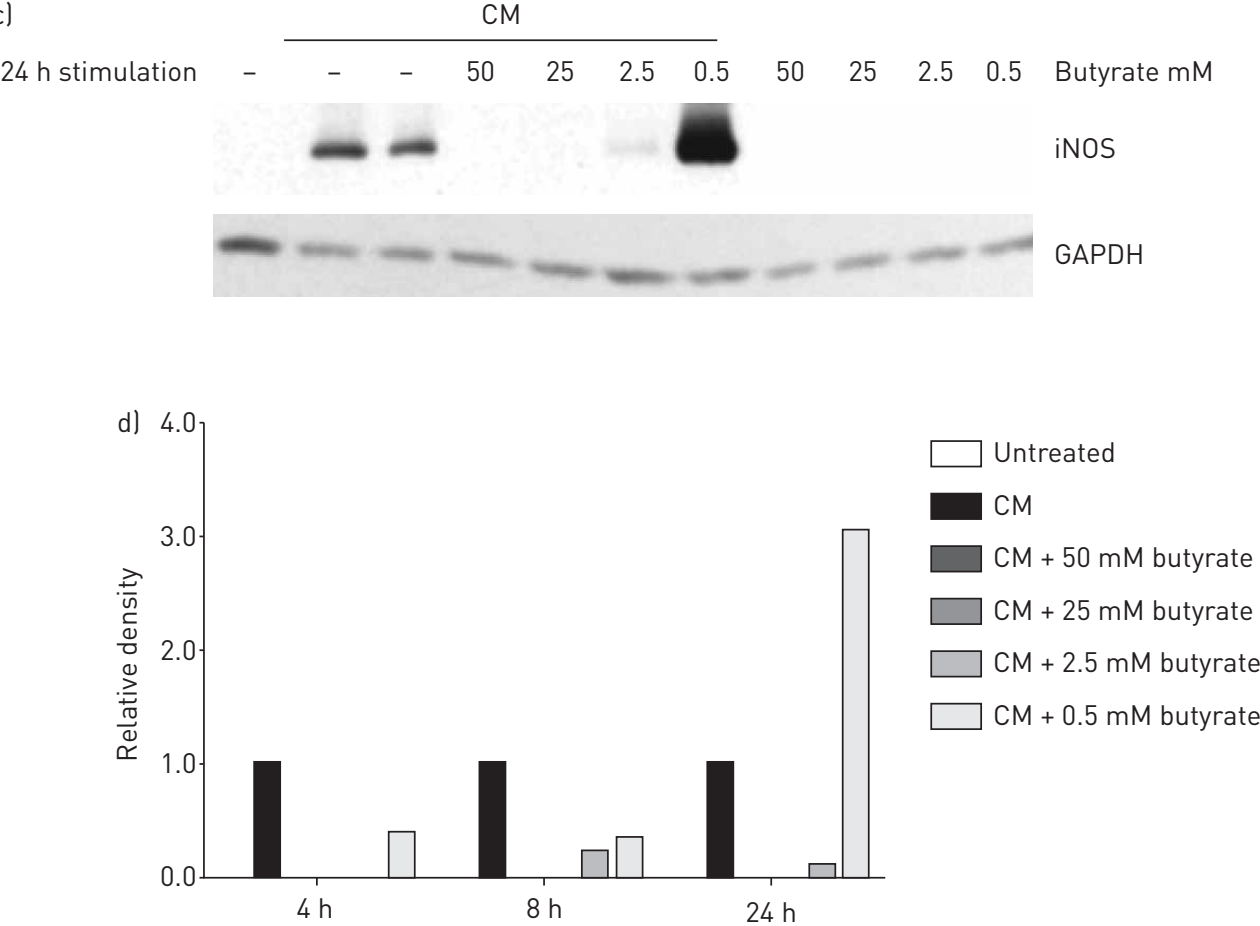

FIGURE 5 Short-chain fatty acids (SCFAs) modulate inducible nitric oxide synthase (iNOS) expression in A549 cells. iNOS and glyceraldehyde 3-phosphate dehydrogenase (GAPDH) expression in A549 cells pre-incubated for $1 \mathrm{~h}$ with different concentrations of butyrate $(0.5 \mathrm{mM}, 2.5 \mathrm{mM}, 25 \mathrm{mM}$ and $50 \mathrm{mM})$ and stimulated with or without cytomix (CM) (10 ng. $\mathrm{mL}^{-1}$ each of interferon- $\gamma$, tumour necrosis factor- $\alpha$ and interleukin-1 $\beta$ ) for a) $4 \mathrm{~h}$, b) $8 \mathrm{~h}$ and c) $24 \mathrm{~h}$. Data are presented as representative Western blots or d) quantification of at least two independent experiments performed in duplicate. Samples and controls for each time point were run on the same gel. Fold change relative to cytokine stimulation alone $(\mathrm{CM})$ is displayed.

SCFAs alter growth of $\mathrm{P}$. aeruginosa

Psuedomonas aeruginosa is a facultative anaerobe, and can produce SCFAs [22]. Since the presence of SCFAs may influence bacterial metabolism, we asked whether SCFAs affect the growth of P. aeruginosa. 
a)
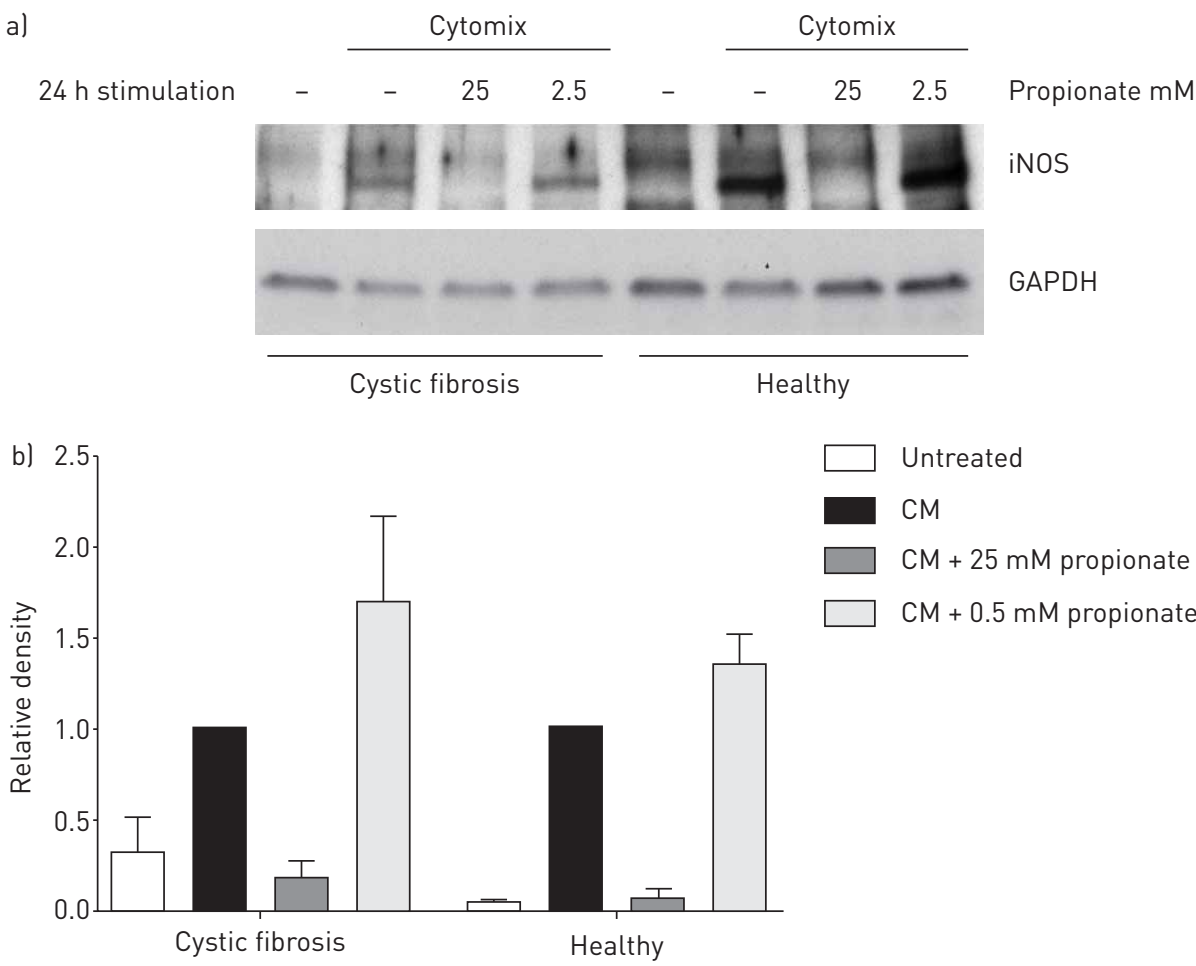

Untreated

$\mathrm{CM}$

$\mathrm{CM}+25 \mathrm{mM}$ propionate

$\mathrm{CM}+0.5 \mathrm{mM}$ propionate

FIGURE 6 Short-chain fatty acids modulate inducible nitric oxide synthase (iNOS) expression in primary epithelial cultures. a) Representative Western blot of iNOS and glyceraldehyde 3-phosphate dehydrogenase (GAPDH) expression in primary cystic fibrosis or healthy control epithelial cells pre-incubated for $1 \mathrm{~h}$ with different concentrations of propionate $(0.5 \mathrm{mM}$ and $25 \mathrm{mM})$ and stimulated with or without cytomix (CM) $\left(10 \mathrm{ng} \cdot \mathrm{mL}^{-1}\right.$ each of interferon- $\gamma$, tumour necrosis factor- $\alpha$ and interleukin-1 $\beta$ ) for $24 \mathrm{~h}$. b) Quantification of three donors for cystic fibrosis and healthy cultures. Data are presented as mean \pm SEM fold change relative to cytokine stimulation alone (CM). Samples and controls were run on the same gel.

In these in vitro experiments we observed a dichotomous concentration-dependent effect of SCFAs on bacterial growth. At high concentrations, propionate (fig. 7), acetate and butyrate (online supplementary figs S3 and S4) inhibited $P$. aeruginosa growth over $\geqslant 6 \mathrm{~h}$. In contrast, we saw that low concentrations of SCFAs resulted in enhanced bacterial growth, particularly during the mid-log phase of growth. Cystic fibrosis airway secretions have been shown to have a slightly acidic $\mathrm{pH}$ [23], although this observation is controversial [24]. As SCFAs have a higher proportion of protonated species in acidic conditions, their diffusion through membranes is greatly enhanced [7]. Therefore we also tested bacterial growth conditions at different $\mathrm{pH}$. We found that at decreased $\mathrm{pH}$ there was uniformly lower bacterial growth with SCFA incubation (fig. 7). We used the laboratory P. aeruginosa strain PAO1 for these experiments, and observed comparable results with a clinical isolate PA508 (online supplementary fig. S5 and data not shown).

As the environment of cystic fibrosis sputum is hypoxic, we sought to determine the effect of SCFAs on $P$. aeruginosa growth under such conditions. We observed that SCFAs caused significant growth inhibition, which was amplified at lower $\mathrm{pH}$, and that overall growth was considerably reduced compared to aerobic conditions (online supplementary fig. S6). Collectively, we conclude that SCFAs alter the growth of Pseudomonas strains in a concentration- and $\mathrm{pH}$-dependent manner.

\section{Discussion}

This is the first study to quantitatively assess the presence of SCFAs in the airway secretions of patients with cystic fibrosis. Sputum SCFA concentrations were found in the millimolar range in both clinically stable patients and those presenting with a pulmonary exacerbation. Antibiotic treatment resulted in a decrease in SCFA levels in most, but not all sputum samples, suggesting that bacteria contributed to the SCFA levels in sputum. However, as sputum SCFA levels before treatment for pulmonary exacerbation were not from clinically stable patients, other factors may also contribute to SCFAs in airways. These could include differences in quantitative and qualitative airway microbiology, bacterial metabolic activity, degree of airway inflammation, as well as differences in individual treatments. We then studied the effects of SCFAs on inflammatory responses of cultured airway epithelial cells. While high concentrations of 

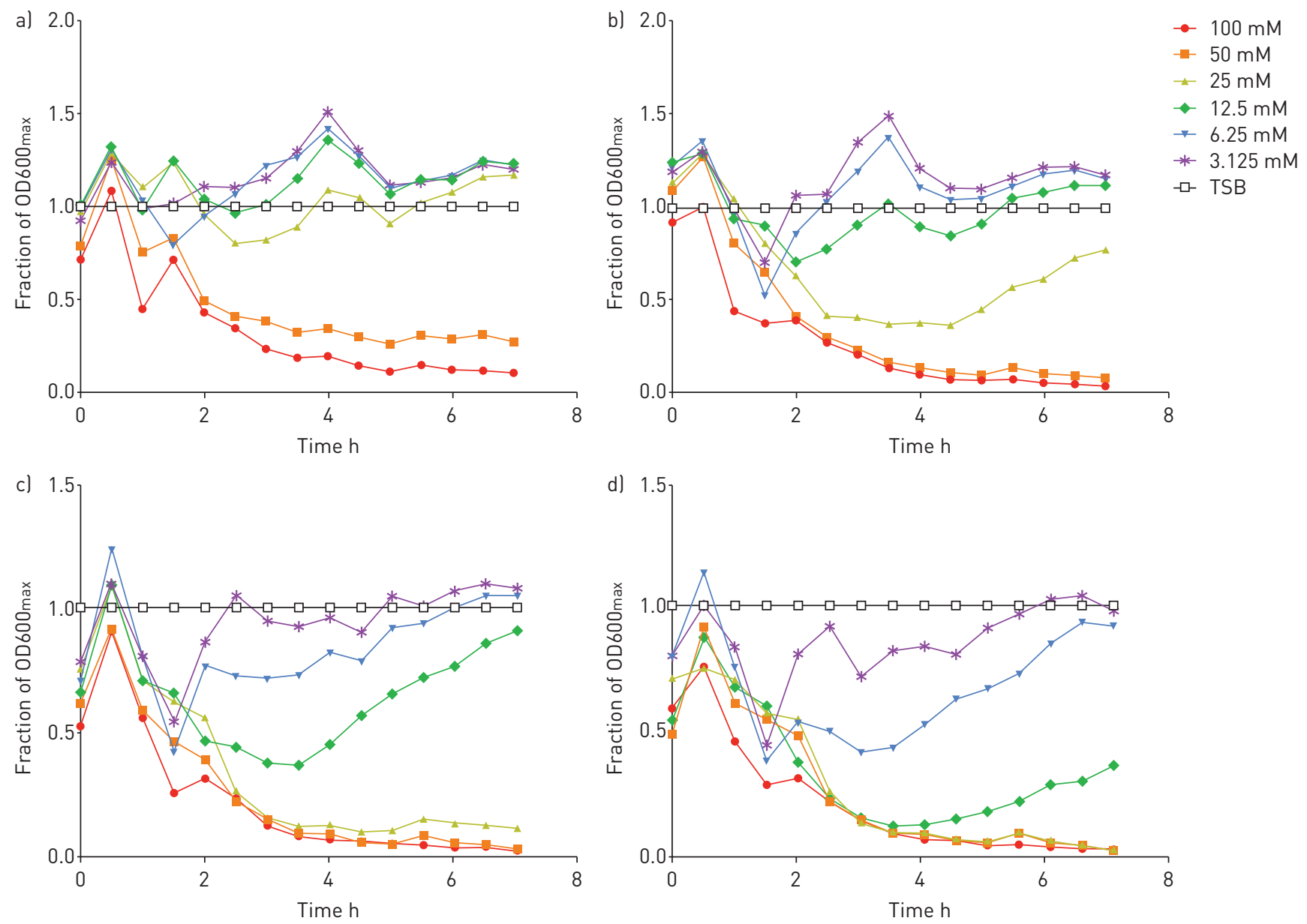

FIGURE 7 Effect of propionate concentration and $\mathrm{pH}$ on the growth of PA01. Pseudomonas aeruginosa PA01 was incubated with propionate at concentrations between $3.125 \mathrm{mM}$ and $100 \mathrm{mM}$ in trypticase soy broth (TSB). pH was adjusted using HCl to al 7.0 ; b) 6.5; c) 6.0; and d) 5.5. Optical density (OD) readings at $600 \mathrm{~nm}$ were normalised to the average OD from TSB alone at each time point. Experiments were performed in duplicate and data are representative of at least two independent experiments.

propionate and butyrate reduced proliferation of A549 cells, they did not influence their viability (online supplementary fig. S7). Our findings show that SCFAs can influence the inflammatory response of airway epithelium and may contribute to differences between normal and cystic fibrosis airway inflammation. We found that high (25-50 mM) concentrations of SCFAs enhanced the induction of inflammatory cytokines such as GM-CSF and IL-6 in A549 and corrCFBE cells, whereas low (0.5-2.5 mM) concentrations of acetate upregulated IL-8 in CFBE but not corrCFBE cells. In primary cell cultures, high $(25 \mathrm{mM})$ concentrations of SCFAs inhibited IL-8 production in non-cystic fibrosis controls, but not in cystic fibrosis airway epithelium, suggesting that SCFAs may contribute to increased IL-8 levels, which are characteristic of cystic fibrosis airway inflammation [17].

Interestingly, earlier studies investigating the effects of butyrate and its derivative 4-phenylbutyrate on pharmacological rescue of F508del-CFTR found, in line with our results, that 4-phenylbutyrate (at 6-10 mM) had a pro-inflammatory effect on CFBE cells (CFBE41o-, also used in this study, and IB3-1 cells) by upregulating IL-8 $[25,26]$. However, in our study there was no correlation between SCFA concentrations at the time of pulmonary exacerbation with IL- 8 or the change in IL- 8 after antibiotic treatment, and no correlation between the change in SCFA concentrations before and after antibiotic treatment with either the change in IL-8 or the levels of IL-8 after antibiotic treatment, illustrating that SCFAs may contribute to but are not the major factor affecting individual IL-8 levels. The SCFA-mediated changes in cytokine production from epithelial cell cultures suggest that SCFAs could lead to impaired resolution of inflammation and exacerbated persistence of neutrophils. This was supported by the significant positive correlation between SCFA concentrations and total neutrophil count in cystic fibrosis sputum. Apart from the effect on cytokine levels, SCFAs can induce chemotaxis in neutrophils, and may thus also have a direct effect on airway neutrophilia [27]. Larger studies are needed to further elucidate the link between SCFAs and airway inflammation in cystic fibrosis. 
The expression of iNOS, an enzyme important for the production of protective reactive nitrogen species, is impaired in cystic fibrosis airway epithelium [21, 28, 29], and NO concentrations in the airways of patients with cystic fibrosis are typically decreased [30,31]. However, antibiotic therapy for acute pulmonary exacerbations can lead to improved iNOS expression [32] and airway NO production $[11,12]$ in cystic fibrosis. Changes in NO metabolism following treatment of exacerbations correlated with improvement in pulmonary function $[10,33]$; consequently, other more direct strategies to improve airway NO production in cystic fibrosis are being investigated $[34,35]$. We show here that SCFAs can affect iNOS expression in a concentration-dependent manner in alveolar and airway epithelial cells in vitro. In A549 cells, normal control and primary cystic fibrosis epithelial cell cultures, low concentrations enhanced cytokine-stimulated iNOS expression. In contrast, high concentrations markedly abrogated iNOS expression in A549 as well as primary control and cystic fibrosis epithelial cells. While it is not clear whether the effect on iNOS expression was direct or mediated by changes in the inflammatory response, SCFAs and their derivatives have previously been shown to directly inhibit activation of nuclear factor- $\kappa \mathrm{B}$, a key regulator of iNOS transcription $[26,36]$. The presence of SCFAs in airways may contribute to the dysregulation of NOS in cystic fibrosis airways, which is supported by our observation that lower concentrations of these bacterial metabolites in sputum of cystic fibrosis patients were predictive for the increase in airway NO after antibiotic therapy in our small cohort.

$P$. aeruginosa is an opportunistic pathogen that frequently leads to chronic cystic fibrosis airways infections. Extending previous studies on SCFA-mediated growth inhibition of Pseudomonas species in vitro [37], we compared the effects of low and high millimolar concentrations of SCFAs and the $\mathrm{pH}$-dependent nature of their effect on P. aeruginosa growth. We found that while high millimolar concentrations impaired growth, in line with earlier reports [37, 38], low millimolar concentrations provided a transient boost in growth during the mid-log phase. P. aeruginosa growth was universally reduced with decreasing $\mathrm{pH}$ in the presence of SCFAs. Furthermore, we confirmed these SCFA effects in microaerobic conditions. It is also conceivable that SCFAs may influence $P$. aeruginosa biofilm formation, but this was not investigated in these studies. Together, our results therefore show that SCFAs act both on the host to aggravate inflammatory responses and on colonising bacteria to increase their growth. These findings may also be of relevance in other anaerobic pulmonary infections such as empyema or lung abscess, where SCFAs are also detectable and correlate with positive anaerobic bacterial cultures [39].

Lately, there has been a surge of interest in SCFAs with the publication of several reports about SCFAs and their role in maintaining colonic regulatory $\mathrm{T}$ (Treg) cells $[8,40]$. These studies showed that germ-free mice, deficient in SCFAs, are also deficient in Tregs and that faecal extracts from germ-free or antibiotic-treated mice poorly induce Foxp $3^{+}$Tregs in vitro. Butyrate was identified as particularly important for generating Tregs and this effect was Ffar2-dependent [8]. The balance between T-helper (Th)17 cells and Tregs is closely linked to inflammation and tolerance in the intestine [41]. As the Th17 pathway primarily evokes a neutrophil response, this is particularly interesting in the context of the prevalent neutrophilia found in cystic fibrosis lung disease $[18,42]$. Given the similarities of the mucosal environments of the gut and the lungs, it will be important to investigate whether SCFAs can modulate the Th17/Treg axis in the cystic fibrosis airways [43]. The cell type-specific expression patterns of FFAR2 and other SCFA receptors in the airways are still unclear, but their elucidation may provide insight into the mechanism of SCFA activity and target cell populations.

In summary, our data suggest that SCFAs in concentrations found in sputum from cystic fibrosis patients exert cystic fibrosis-specific effects on airway epithelium inflammatory responses, induction of iNOS and $P$. aeruginosa growth. Further studies are needed to better characterise the role of these molecules in cystic fibrosis airway inflammation and to understand whether SCFAs in cystic fibrosis sputum can be used as markers of anaerobic bacterial activity or predictors of response to antimicrobial therapy.

\section{Acknowledgements}

We thank Kervan Rivera-Rufner (Dept of Nutritional Sciences, University of Toronto, Toronto, ON, Canada), Darakhshanda Shehnaz and Hailu Huang (Research Institute, The Hospital for Sick Children, Toronto) for excellent technical assistance. We thank David N. Douda (Brigham and Women's Hospital and Harvard Medical School, Boston, MA, USA), Helena Obernolte, Armin Braun and Katherina Sewald (Fraunhofer ITEM, Hannover, Germany) for helpful discussions. We thank Christine Bear (Research Institute, The Hospital for Sick Children) for providing cystic fibrosis epithelial cell lines and Neil B. Sweezey (The Hospital for Sick Children) for providing a clinical isolate of Pseudomonas aeruginosa. Primary human bronchial epithelial cells and cell culture media were provided by the Primary Airway Cell Biobank from the Cystic Fibrosis Translational Research Centre at McGill University (Montreal, QU, Canada), supported by Cystic Fibrosis Canada. We acknowledge Hailey Craig-Barnes at the Analytical Facility for Bioactive Molecules of the Centre for the Study of Complex Childhood Diseases (CSCCD) at the Hospital for Sick Children (Toronto, ON, Canada), for multiplex ELISA services. The CSCCD was supported by the Canadian Foundation for Innovation (Ottawa, ON, Canada). Portions of this manuscript were published as part of the Master's thesis of Peyman Ghorbani and as an abstract at the European Respiratory Society's annual meeting in 2012 (Vienna, Austria). 


\section{References}

1 Ratjen F, Döring G. Cystic fibrosis. Lancet 2003; 361: 681-689.

2 Worlitzsch D, Tarran R, Ulrich M, et al. Effects of reduced mucus oxygen concentration in airway Pseudomonas infections of cystic fibrosis patients. J Clin Invest 2002; 109: 317-325.

3 Lyczak JB, Cannon CL, Pier GB. Lung infections associated with cystic fibrosis. Clin Microbiol Rev 2002; 15: 194-222.

4 Tunney MM, Field TR, Moriarty TF, et al. Detection of anaerobic bacteria in high numbers in sputum from patients with cystic fibrosis. Am J Respir Crit Care Med 2008; 177: 995-1001.

5 Worlitzsch D, Rintelen C, Böhm K, et al. Antibiotic-resistant obligate anaerobes during exacerbations of cystic fibrosis patients. Clin Microbiol Infect 2009; 15: 454-460.

6 Mortensen PB, Clausen MR. Short-chain fatty acids in the human colon: relation to gastrointestinal health and disease. Scand J Gastroenterol Suppl 1996; 216: 132-148.

7 Sellin JH. SCFAs: the enigma of weak electrolyte transport in the colon. News Physiol Sci 1999; 14: 58-64.

8 Smith PM, Howitt MR, Panikov N, et al. The microbial metabolites, short-chain fatty acids, regulate colonic Treg cell homeostasis. Science 2013; 341: 569-573.

9 Al-Saleh S, Dell SD, Grasemann H, et al. Sputum induction in routine clinical care of children with cystic fibrosis. J Pediatr 2010; 157: 1006-1011.

10 Grasemann H, Al-Saleh S, Scott JA, et al. Asymmetric dimethylarginine contributes to airway nitric oxide deficiency in patients with cystic fibrosis. Am J Respir Crit Care Med 2011; 183: 1363-1368.

11 Gaston B, Ratjen F, Vaughan JW, et al. Nitrogen redox balance in the cystic fibrosis airway: effects of antipseudomonal therapy. Am J Respir Crit Care Med 2002; 165: 387-390.

12 Jaffé A, Slade G, Rae J, et al. Exhaled nitric oxide increases following admission for intravenous antibiotics in children with cystic fibrosis. J Cyst Fibros 2003; 2: 143-147.

13 Böcker U, Nebe T, Herweck F, et al. Butyrate modulates intestinal epithelial cell-mediated neutrophil migration. Clin Exp Immunol 2003; 131: 53-60.

14 Stempelj M, Kedinger M, Augenlicht L, et al. Essential role of the JAK/STAT1 signaling pathway in the expression of inducible nitric-oxide synthase in intestinal epithelial cells and its regulation by butyrate. J Biol Chem 2007; 282: 9797-9804.

15 Maslowski KM, Vieira AT, $\mathrm{Ng} \mathrm{A}$, et al. Regulation of inflammatory responses by gut microbiota and chemoattractant receptor GPR43. Nature 2009; 461: 1282-1286.

16 Macia L, Thorburn AN, Binge LC, et al. Microbial influences on epithelial integrity and immune function as a basis for inflammatory diseases. Immunol Rev 2012; 245: 164-176.

17 Eickmeier O, Huebner M, Herrmann E, et al. Sputum biomarker profiles in cystic fibrosis (CF) and chronic obstructive pulmonary disease (COPD) and association between pulmonary function. Cytokine 2010; 50 : 152-157.

18 Tiringer K, Treis A, Fucik P, et al. A Th17- and Th2-skewed cytokine profile in cystic fibrosis lungs represents a potential risk factor for Pseudomonas aeruginosa infection. Am J Respir Crit Care Med 2013; 187: 621-629.

19 Cozens AL, Yezzi MJ, Chin L, et al. Characterization of immortal cystic fibrosis tracheobronchial gland epithelial cells. Proc Natl Acad Sci USA 1992; 89: 5171-5175.

20 Shuto T, Furuta T, Oba M, et al. Promoter hypomethylation of Toll-like receptor-2 gene is associated with increased proinflammatory response toward bacterial peptidoglycan in cystic fibrosis bronchial epithelial cells. FASEB J 2006; 20: 782-784.

21 Meng QH, Springall DR, Bishop AE, et al. Lack of inducible nitric oxide synthase in bronchial epithelium: a possible mechanism of susceptibility to infection in cystic fibrosis. J Pathol 1998; 184: 323-331.

22 Moss CW, Dees SB. Cellular fatty acids and metabolic products of Pseudomonas species obtained from clinical specimens. J Clin Microbiol 1976; 4: 492-502.

23 Pezzulo AA, Tang XX, Hoegger MJ, et al. Reduced airway surface $\mathrm{pH}$ impairs bacterial killing in the porcine cystic fibrosis lung. Nature 2012; 487: 109-113.

24 McShane D, Davies JC, Davies MG, et al. Airway surface pH in subjects with cystic fibrosis. Eur Respir J 2003; 21: 37-42.

25 Rubenstein RC, Egan ME, Zeitlin PL. In vitro pharmacologic restoration of CFTR-mediated chloride transport with sodium 4-phenylbutyrate in cystic fibrosis epithelial cells containing delta F508-CFTR. J Clin Invest 1997; 100: 2457-2465.

26 Roque T, Boncoeur E, Saint-Criq V, et al. Proinflammatory effect of sodium 4-phenylbutyrate in deltaF508-cystic fibrosis transmembrane conductance regulator lung epithelial cells: involvement of extracellular signal-regulated protein kinase 1/2 and c-Jun-NH2-terminal kinase signaling. J Pharmacol Exp Ther 2008; 326: 949-956.

27 Vinolo MA, Ferguson GJ, Kulkarni S, et al. SCFAs induce mouse neutrophil chemotaxis through the GPR43 receptor. PLoS One 2011; 6: e21205.

28 Kelley TJ, Drumm ML. Inducible nitric oxide synthase expression is reduced in cystic fibrosis murine and human airway epithelial cells. J Clin Invest 1998; 102: 1200-1207.

29 Grasemann H, Ratjen F. Nitric oxide and L-arginine deficiency in cystic fibrosis. Curr Pharm Des 2012; 18: 726-736.

30 Grasemann H, Michler E, Wallot M, et al. Decreased concentration of exhaled nitric oxide (NO) in patients with cystic fibrosis. Pediatr Pulmonol 1997; 24: 173-177.

31 Zheng S, Xu W, Bose S, et al. Impaired nitric oxide synthase-2 signaling pathway in cystic fibrosis airway epithelium. Am J Physiol Lung Cell Mol Physiol 2004; 287: L374-L381.

32 Wooldridge JL, Deutsch GH, Sontag MK, et al. NO pathway in CF and non-CF children. Pediatr Pulmonol 2004; 37: 338-350.

33 Grasemann H, Schwiertz R, Matthiesen S, et al. Increased arginase activity in cystic fibrosis airways. Am J Respir Crit Care Med 2005; 172: 1523-1528.

34 Grasemann H, Tullis E, Ratjen F. A randomized controlled trial of inhaled L-arginine in patients with cystic fibrosis. J Cyst Fibros 2013; 12: 468-474.

35 Mehl A, Ghorbani P, Douda D, et al. Effect of arginase inhibition on pulmonary L-arginine metabolism in murine Pseudomonas pneumonia. PLoS One 2014; 9: e90232.

36 Yin L, Laevsky G, Giardina C. Butyrate suppression of colonocyte NF- $\mathrm{KB}$ activation and cellular proteasome activity. J Biol Chem 2001; 276: 44641-44646. 
37 Levison ME. Effect of colon flora and short-chain fatty acids on growth in vitro of Pseudomonas aeruginosa and Enterobacteriaceae. Infect Immun 1973; 8: 30-35.

38 Sallam KI. Antimicrobial and antioxidant effects of sodium acetate, sodium lactate, and sodium citrate in refrigerated sliced salmon. Food Control 2007; 18: 566-575.

39 Hunter JV, Chadwick M, Hutchinson G, et al. Use of gas liquid chromatography in the clinical diagnosis of anaerobic pleuropulmonary infection. Br J Dis Chest 1985; 79: 1-8.

40 Atarashi K, Tanoue $\mathrm{T}$, Oshima $\mathrm{K}$, et al. Treg induction by a rationally selected mixture of Clostridia strains from the human microbiota. Nature 2013; 500: 232-236.

41 Littman DR, Rudensky AY. Th17 and regulatory T cells in mediating and restraining inflammation. Cell 2010; 140: 845-858.

42 Iannitti RG, Carvalho A, Cunha $\mathrm{C}$, et al. Th17/Treg imbalance in murine cystic fibrosis is linked to indoleamine 2,3-dioxygenase deficiency but corrected by kynurenines. Am J Respir Crit Care Med 2013; 187: 609-620.

43 Kushwah R, Gagnon S, Sweezey NB. Intrinsic predisposition of naïve cystic fibrosis T cells to differentiate towards a Th17 phenotype. Respir Res 2013; 14: 138. 